\title{
Flat sheet of Zinc material as a filter for gamma photons in Tc-99mm SPECT imaging
}

\author{
Nazifah Abdullah ${ }^{1,2 *}$, Inayatullah Shah Syed ${ }^{1}$, Ahmad Zakaria ${ }^{3}$ \\ ${ }^{1}$ Kulliyyah of Allied Health Sciences, International Islamic University Malaysia, 25200 Kuantan, Pahang, Malaysia, Tel: +60 (16) \\ 7153781 Fax: +60 (9) 5716776 \\ ${ }^{2}$ Faculty of Health Science, Universiti Sultan Zainal Abidin (UniSZA), Gong Badak Campus, 21300 Kuala Terengganu, Terengganu, \\ Malaysia, Tel:+60(19)9555507, Fax: +60(9) 6687896 \\ ${ }^{3}$ School of Health Sciences, Universiti Sains Malaysia, 16150 Kubang Kerian, Kota Bharu, Kelantan, Malaysia, Tel: +60(12)3087278,
} Fax: $+60(9) 7677515$

\begin{abstract}
Purpose of the Study: Scattered gamma photons in single photon emission computed tomography (SPECT) is one of the main issues that degrade the image quality. There are several types of scatter correction methods. However, none of the technique is applied in routine clinical Tc-99m SPECT imaging. Therefore, Zinc (Zn) material filter was constructed for pre-filtration of scattered gamma photons in Tc-99m single photon emission computed tomography (SPECT).

Materials and Methods: Tc-99m radioactivity was administered into the Carlson's phantom. Planar and tomographic data were acquired with and without hot regions insert, respectively, with and without material filter. Scatter to non-scatter ratio was measured from the photopeak region of Tc-99m spectra. Tomographic images were generated using filtered back projection method. Contours on images were drawn with ImageJ software. Images obtained with and without material filter were analysed in terms of detectability of hot regions.

Results: Remarkable decrease $(\approx 21 \%)$ in the ratio of scattered to non-scattered gamma photons with material filter was achieved. Detectability of smaller hot regions with material filter was enhanced. In terms of sizes of all hot regions, material filter results appear closer to the original sizes.

Conclusion: Material filter technique could be applied in Tc-99m clinical SPECT, while organ phantom studies are needed.
\end{abstract}

\section{Keywords: Material Filter, Scatter Correction, SPECT Image Quality, Contrast}

\section{Introduction}

Single photon emission imaging; planar and tomography have played a significant role in the diagnosis of diseases. This imaging modality provides important functional information of the organ / body part under investigation. However, detection of scattered gamma photons in image data limits the performance of the technique. Due to poor energy resolution of imaging equipment, up to $40 \%$ of scattered gamma photons are detected in the standard energy window ( $20 \%$ centered at $140 \mathrm{keV}$ ) data which degrades the quality of reconstructed image [1-2]. In order to overcome the scattered gamma photons issue, researchers have developed a number of scatter correction techniques, as mentioned in a review on scatter correction techniques by $[1,3]$ such as; use of an effective attenuation coefficient value, window subtraction, and computational techniques. Unfortunately, in patient studies none of the scatter correction techniques are being used, primarily due to implementation issues, high cost and unreliable outcome [1].

Another approach may be termed as unconventional pre-filtering; material filter technique, which was introduced by $[4,5]$ in Tc-99m planar imaging. This technique is developed on the fact that scattered gamma photons possess low energy as compared to nonscattered. Thus, preferentially more scattered gamma photons are absorbed by the material filter due to their comparatively less energy. The material filter either can be installed over the outer surface of the gamma camera collimator or between the collimator and the scintillation detector. Therefore, a significant fraction of the scattered gamma photons would be pre-filtered from the image data. Moreover, this technique may be called as the online scatter reduction technique. Recently, thin flat sheets of Tin (Sn) [6] and Copper (Cu) [7] materials were used as absorbers of scattered gamma photons in Tc-99m SPECT. Tin and Copper filters show reduction in scatter fraction and improvement in image quality with material filters as compared to unfiltered data images. However, a thin flat sheet of $\mathrm{Sn}$ is fragile, and $\mathrm{Cu}$ has the tendency to easily bend. This makes it difficult to keep the filters safe from cracks and flat for use by mounting on the outer surface of gamma camera collimator. Therefore, the aim of this study is to construct a material filter from a flat sheet of another material; Zinc (Zn) to preferentially absorb some fraction of scattered gamma photons before reaching the scintillation detector - $\mathrm{NaI}(\mathrm{Tl})$ - of the gamma camera. This filter has been constructed for Tc-99m (140 keV) considering the most common use as a radionuclide in single photon emission computed tomography. In addition, earlier studies show the effect of $\mathrm{Zn} 0.2 \mathrm{~mm}$ thick material filter on spatial resolution, system uniformity and system volume sensitivity, [8] contrast, signal-to-noise ratio (SNR), detectability of Tc-99m SPECT hot and cold regions [9]. Improvement in spatial resolution, contrast (smaller hot and cold regions), SNR and detectability was achieved. In this work, measurement of scatter to non-scatter ratio from the photo peak region $(126-164 \mathrm{keV})$ of Tc-99m spectra and image quality of transverse images of hot regions has been 
investigated. It is expected that; the use of zinc material filter can play a vital role in enhancing the quality of Tc-99m SPECT images.

\section{Material and methods}

Several materials were considered based on certain material characteristics, such as the atomic number, thickness and purity. In this regard, Aluminum (Al), Ferrum (Fe), Nickel (Ni), Copper $(\mathrm{Cu})$ and Zinc $(\mathrm{Zn})$ were thought-out to be suitable materials for the construction of a material filter. Theoretical calculations were made for 0.1 to $0.4 \mathrm{~mm}$ thicknesses with $0.1 \mathrm{~mm}$ increment in order to establish the reduction fraction of various gamma photon energies [8]. Based on the calculations of percent attenuation for a range of gamma photon energies, the thicknesses 0.1 and $0.2 \mathrm{~mm}$ of $\mathrm{Zn}$ material were found useful for Tc-99m $(140 \mathrm{keV})$ radionuclide imaging [8]. A flat sheet of $\mathrm{Zn}$ material $0.2 \mathrm{~mm}$ thick was used $(0.1 \mathrm{~mm}$ was not available). The accuracy of thickness of the sheet was measured by an electronic Vernier Caliper. The reading was repeated for three times, +/- $0.01 \mathrm{~mm}$ error in the thickness was found. Constructed $\mathrm{Zn}$ material filter is shown in Figures 1 and 2. Furthermore, in order to make sure that there are no cracks in the sheet and verify the purity and presence of other elements in the material of filter, SEM and EDX analyses were performed.

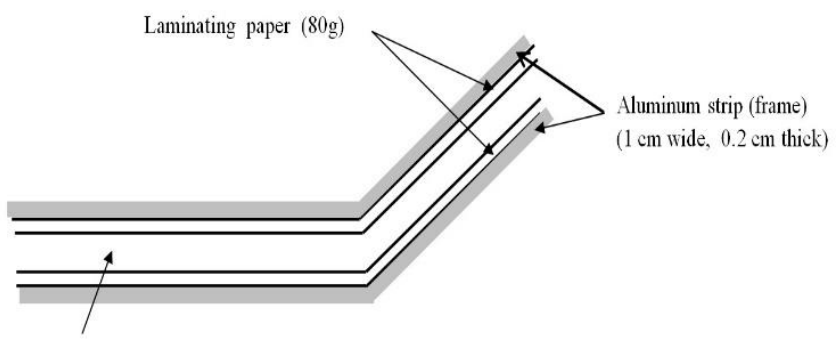

Zine $(0.2 \mathrm{~mm})$

Fig. 1: Show the schematic diagram of $\mathrm{Zn} 0.2 \mathrm{~mm}$ thick material filter.

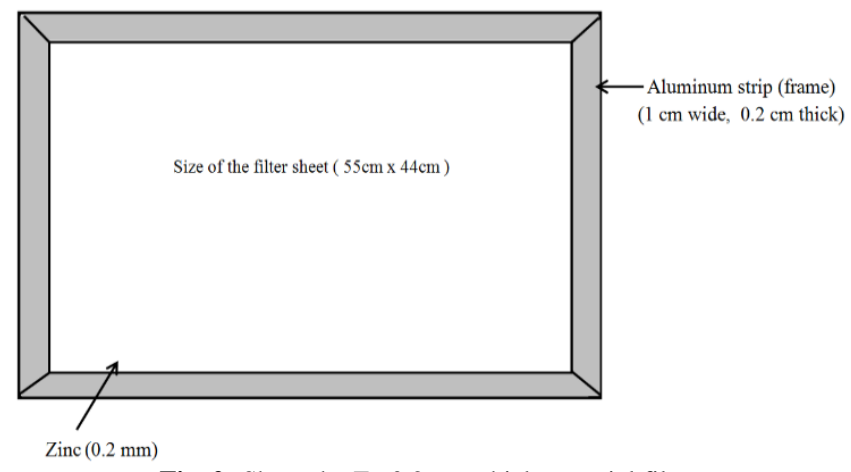

Fig. 2: Show the Zn $0.2 \mathrm{~mm}$ thick material filter.

The surface morphology of filter material can be observed from SEM image as shown in Figure 3. The EDX spectrum of $\mathrm{Zn} 0.2$ $\mathrm{mm}$ (Figure 4), shows an intense peak located at $\sim 1 \mathrm{keV}$, this reflects the presence of $\mathrm{Zn}$ element in sample and the table embedded depicts the weight percent of $\mathrm{Zn}$ including other elements $\mathrm{(Cu}$ and $\mathrm{O})$.

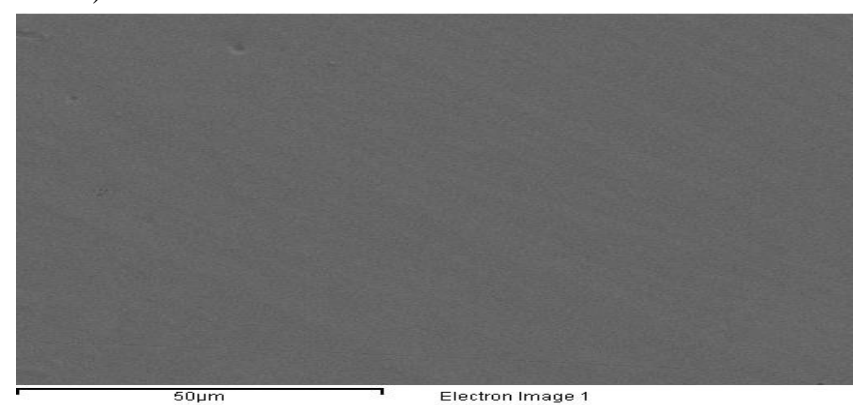

Fig. 3: Illustrate the surface morphology of Zn $0.2 \mathrm{~mm}$ material filter applying scanning electron microscopy.

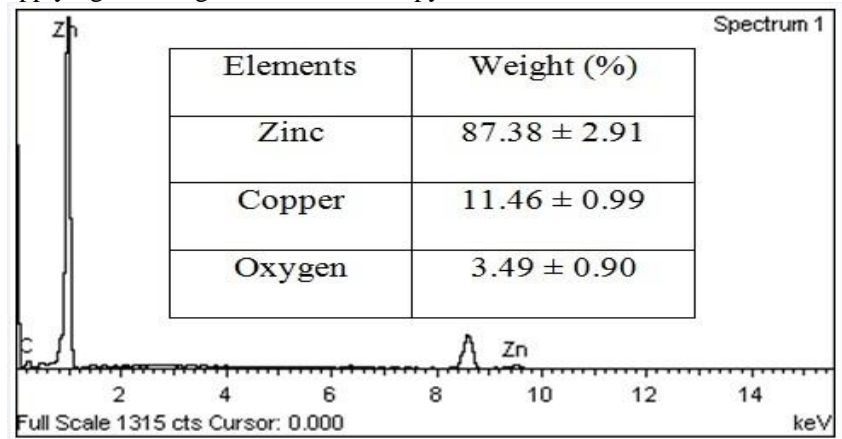

Fig. 4: Depicts the Energy Dispersive X-Ray spectrum of $\mathrm{Zn}$ material and table imbedded show the weight percent of $\mathrm{Zn}, \mathrm{Cu}$ and $\mathrm{O}$ elements.

Cylindrical source tank of Carlson's phantom filled with clean water; injecting $15.5 \mathrm{mCi}$ of Tc-99m radioactivity was scanned. A visual indicator (i.e., ink) was added into the source tank to observe the uniform mixing of radioactivity. Air bubbles were removed completely from the tank. Furthermore, the cylindrical source tank was set to rest for about 30 minutes before scanning. The Philips ADAC Forte dual head gamma camera was used. The cylindrical source tank was positioned on the scanning table, parallel to the axis of rotation at the centre of gamma camera's field of view (FOV) and closer to the low energy high resolution collimator (LEHR) at a distance of $2 \mathrm{~cm}$. For plotting the spectra of Tc-99m, count rate was recorded by setting various energy windows from $54 \mathrm{keV}$ up to $160 \mathrm{keV}$, with an increment of $8 \mathrm{keV}$ (i.e., $54-160,62-160, \ldots \ldots$, and $156-160 \mathrm{keV}$ ). Each reading was repeated for three times. For each window with and without material filter, the duration of data collection was maintained at 5 seconds. Count rate was corrected for radioactive decay. The ratio of scattered to non-scattered photons was obtained by measuring the area under the photopeak using the trapezoidal approximation/area method [10].

For SPECT data collection, Carlson's phantom with hot regions insert was used. The procedure for preparation of phantom was similar to as that decsribed earlier. A hot regions insert, comprised of nine pairs of different diameters $(4.7,5.9,7.3,9.2,11.4,14.3$, 17.9, 22.4 and $29.9 \mathrm{~mm}$ ), eight pairs in a "V" shape positioned at various locations (Figure 5) was placed into the cylindrical tank. Holes simulating hot regions are drilled across the thick solid block of transparent acrylic material. The quantity of radioactive material (Tc-99m) administered into the phantom was $21 \mathrm{mCi}$. Data were collected by Philips ADAC Forte dual head gamma camera with $\mathrm{Zn} 0.2 \mathrm{~mm}$ material filter attached on the outer surface of LEHR collimator and without material filter. The matrix size used was 64 x 64 x 16. Standard energy window (20\%) centred at $140 \mathrm{keV}$ was adjusted. The phantom was positioned on the patient table at equal distances from both detectors. Gamma camera was rotated clockwise 3600 , in oreder to collect image data for all 120 projections. As compared to planar imaging, in SPECT the distance of the cylindrical tank to the detector was little more, to ensure that there was no collision between the heads of gamma camera and the table top. Count rate per projection was maitained about same for with and without material filter data. In this study, 120 projections were taken instead of 64 projections as recommended by IAEA.[11] Also stated by[12] that high number of projections could prevent formation of the star artefact and blurring of the image. For reconstruction of images, all acquired SPECT image data (with and without material filter) were processed by using filtered back projection technique. A digital filter (Butterworth) was used by choosing a 0.35 cycles/cm cut off frequency and order 5. Chang's method for attenuation compensation was applied by selecting $0.12 / \mathrm{cm}$ linear attenuation coefficient $[13,14]$. 


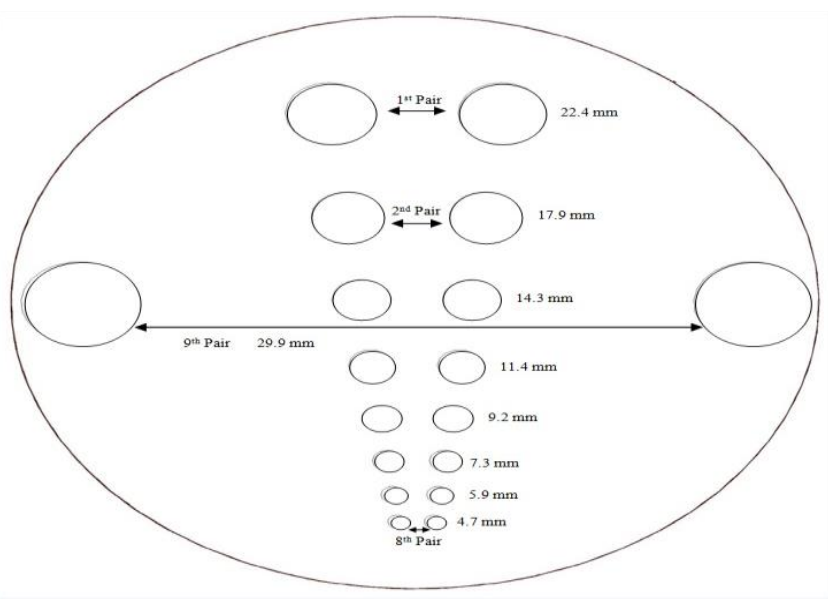

Fig. 5: Cross-sectional view of R. A. Carlson's phantom

\section{Results}

Figure 6 shows the ratio of scattered to non-scattered gamma photons with the use of $\mathrm{Zn} 0.2 \mathrm{~mm}$ and without and material filter. A remarkable decrease $(\approx 21 \%)$ in the scattered to non-scattered ratio with material filter has been achieved.

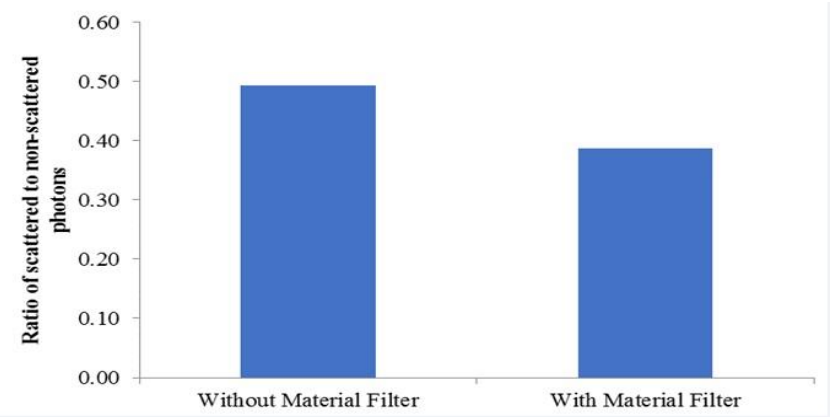

Fig. 6: Scattered to non-scattered gamma photon ratio in $126-154 \mathrm{keV}$ energy window with and without material filter

Scanning electron microscopy is helpful in characterizing the texture or surface morphology of solid materials, e.g., surface defects, stains and deposits of impurities on the surface. As shown in Figure 3 SEM image revealed that the sample of $\mathrm{Zn} 0.2 \mathrm{~mm}$ material has a smooth surface and free from defects and cracks. This type of analysis of material filter was important, as it has an impact on the adverse effect on the absorption and transmission of gamma photons emanating from the object/phantom. Furthermore, the EDX method provides quantitative information about the composition of the material under investigation. Figure 4 was achieved by EDX method in which an intense peak reflects the dominancy of $\mathrm{Zn}$ in the specimen over the other elements and was further proved by the highest weight percent as shown in table imbedded in Figure 4. Results of both tests SEM and EDX are suggestive that $\mathrm{Zn}$ material is a suitable candidate for the construction and implementation for pre-filtering of scattered gamma photons from the projection data in Tc-99m SPECT.

SPECT images of hot regions with and without material filter, as shown in Figure 7 were analyzed visually. For this purpose, good quality transverse slices of hot region images were chosen by experienced staffs who are involved in analyzing the diagnostic images at the centre where data were collected. The same transverse slice numbers of hot region images with and without material filter were selected to avoid any inconsistency in image analysis. To study the effects of material filter on image quality, contours on hot region images were obtained by using ImageJ software [15]. The Image quality parameters being studied were hot regions detectability and impact of material filter on the apparent size of hot regions.

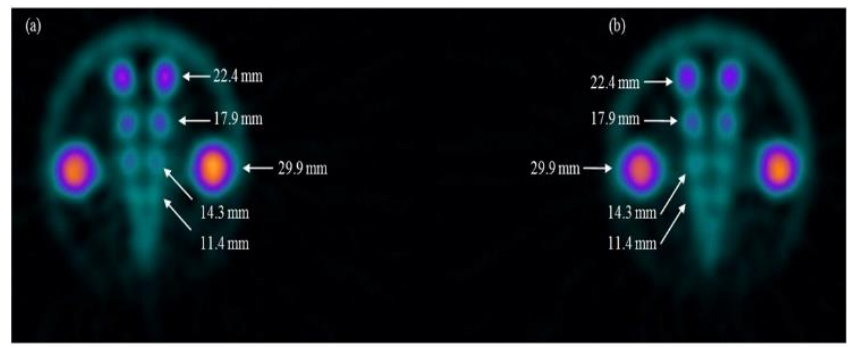

Fig. 7: Transverse images of hot regions (a) without material filter and (b) with material filter

The hot region image shown in Figure 8 was qualitatively evaluated. Contour and visual analysis show a significant improvement in the image quality with material filter. Four hot region pairs are clearly seen, and apparent size of hot regions is smaller with material filter as compared to without material filter. The clear space (no connectivity) between the fourth pair of hot regions with material filter can be visualized as compared to without material filter.

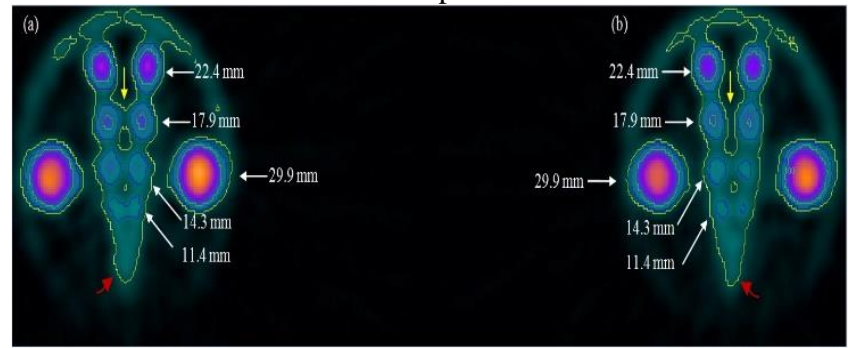

Fig. 8: Transverse images of hot regions (a) without material filter and (b) with material filter. Contour on hot regions plotted using ImageJ software

\section{Discussion}

SPECT imaging inherits numerous problems, such as; poor energy resolution and detection of scattered gamma photons in image data. Studies show that approximately $40 \%$ scattered gamma photons are still present in the data collected within the standard energy window $(126-154 \mathrm{keV})$ for Tc-99m radionuclide $[2,16]$. About $80 \%$ of the total scattered gamma photons is present in the lower part of the photopeak window (below $140 \mathrm{keV}$ ) [17]. Furthermore, literature shows that not only the single scatter but the multiple scattered gamma photons which make about twenty percent of the total scatter are also detected into the standard energy window $(20 \%)$ centered at $140 \mathrm{keV}[1]$.

Results of scatter to non-scatter ratio study show that $\mathrm{Zn} 0.2 \mathrm{~mm}$ material filter technique is effective and capable of removing a remarkable fraction of object scattered (single + multiple) gamma photons before they reach the detector of gamma camera. Material filter preferentially absorbs the scattered gamma photons from the photopeak region $(126-154 \mathrm{keV})$ which have low energy, whether they are single or multiple scattered as compared to nonscattered photons. Therefore, the image data could be considered as free from a significant number of scatters. Furthermore, image reconstruction techniques amplify the noise signals; therefore, in routine clinical studies unwanted data are processed for generation of images. Therefore, material filtered data would provide improved quality of reconstructed images. In clinical Tc-99m SPECT studies, inclusion of scattered photons which in principle carry false positional information lead to the degradation of image quality, if not removed from the projection data. Hence, pose the difficulties in accurate diagnosis of diseases.

In clinical SPECT, best quality images are required to extract information which is essential for the accurate diagnosis of the disease. Scatter radiation degrade the image resolution, thus, accurate diagnostic information from the reconstructed image of the scanned object is not extractable. Moreover, in SPECT smaller hot regions are most affected by scattered gamma photons as compared to large sized regions. It is always difficult to detect small regions/lesions in patient's body; this scenario restricts the early 
detection of disease. Therefore, it becomes hard to provide best possible services to patients.

Images obtained from material filtered data enhanced hot regions detectability (Figure $8 \mathrm{~b}$ ), where smaller $(11.4 \mathrm{~mm}$ diameter) hot regions are visible as separate regions as compared to without material filter (Figure 8a). Furthermore, gamma photons (scatter plus non-scatter) travel outward in all directions from hot regions towards the background, where scatter deviate from its point of emission thus carry false positional information. Hence, hot regions appear to be larger than their actual size in an image. Whereas, material filter results (contours) show that, the apparent size of hot regions is smaller in the image as shown in Figure $8 \mathrm{~b}$. Therefore, the size of hot regions can be assumed closer to that the actual size. In addition, material filtered images reflect the removal of scattered gamma photons from the space between the hot region pairs as indicated by yellow arrow in Figure $8 \mathrm{~b}$ as compared to without material filter (Figure 8a). Moreover, the red arrow shows the narrow/sharp end of the shape "V" of eight hot region pairs which cannot be seen as that in the unfiltered image. Thus, material filter results strongly indicate pre-filtration of scattered gamma photons from the projection data. These results are consistent with the findings of earlier studies conducted by [7,9,18-19] using different type of material filters for removal of scattered gamma photons from the Tc-99m SPECT image data.

\section{Conclusion}

In conclusion, a flat sheet of $\mathrm{Zn} 0.2 \mathrm{~mm}$ thick material filter absorbs a considerable amount of object scattered gamma photons prior to detection by the gamma camera. Hot region detectability, particularly small regions was enhanced. In terms of sizes of all hot regions, material filter provided results which appear closer to the original sizes. The results of the material filter, suggest the use of $\mathrm{Zn} 0.2 \mathrm{~mm}$ in clinical Tc-99m SPECT imaging subject to the organ phantom studies and clinical trials.

\section{References}

[1] Hutton BF, Buvat I, Beekman FJ. Review and current status of SPECT scatter correction. Phys Med Biol 2011;56: R85-R112.

[2] Cherry SR, Sorenson JA, Phelps ME. Physics in Nuclear Medicine, 4th ed Elsevier Saunders: Philadelphia; 2012.

[3] Shidahara M, Watabe H, Kim KM, Kato T, Kawatsu S, Kato R. et al. Development of a practical image-based scatter correction method for brain perfusion SPECT: comparison with the TEW method. Eur J Nucl Med Mol Imaging 2005;32: 1193-98.

[4] Pillay M, Shaprio B, Cox PH. The effect of an alloy filter on gamma camera images. Eur J Nucl Medicine 2005;12: 293-96.

[5] Pillay M, Manon RS. Filter for Gamma Ray Camera. US Patent, 4,852,142; 1989 .

[6] Sayed IS. Use of a Tin (Sn) Flat Sheet as a Material Filter for Reduction of Scattered Gamma Photons and Enhancement of Cold Regions Image Quality in Tc-99m SPECT. International Journal of Computing Academic Research 2016;5: 110-21.

[7] Sayed IS, Siti Zawani H. Effects of Copper $0.127 \mathrm{~mm}$ Thick Flat Sheet on Uniform and Hot Region Images as a Material Filter for Scatter Correction in Tc-99m SPECT. International Journal of Computing Academic Research 2016;5: 143-49.

[8] Nazifah A, Norhanna S, Shah SI, Zakaria A. Effect of different thickness of material filter on Tc-99m spectra and performance parameters of gamma camera. Journal of Physics: Conference Series 2014;546: 1- 6 .

[9] Zakaria NN. BSc Dissertation, International Islamic University Malaysia; 2013.

[10] Ogawa K, Harata Y, Ichihara T, Kubo A, Hashimoto S. A practical method for position dependent Compton scatter correction in single photon emission CT A practical method for position dependent Compton scatter correction in single photon emission CT. IEEE Trans Med Imaging 1991;10: 408-12.

[11] International Atomic Energy Agency, IAEA Human Health Series No. 6, IAEA, Vienna; 2009.
[12] Lyra M, Ploussi A, Rouchota M, Synefia S. Filters in 2D and 3D Cardiac SPECT Image Processing. Cardiol Res Pract 2014; 2014: 963264.

[13] Jaszczak RJ, Floyd CE, Coleman RE. Scatter compensation techniques for SPECT. IEEE Trans. Nuclear Sci 1985;32: 786-93.

[14] Harris CC, Greer KL, Jaszczak RJ, Floyd CEJ, Fearnow EC, Coleman RE. Technitium-99m attenuation coefficients in water filled phantom determined with gamma cameras. Med Phys 1984;11: 681-85.

[15] Rasband WS. ImageJ. United States National Institutes of Health, Bethesda, Maryland, USA; 1997 - 2018.

[16] Larsson SA, Axelsson B, Dahl CM, Msaki P. The use of 1-D and 2D scatter deconvolution techniques for contrast enhancement and quantification in SPECT. J Nucl Medicine 1986;27: 884.

[17] Kojima M, Masanori, Mutsumasa T. Experimental analysis of scattered photons in Tc-99m imaging with a gamma camera. Ann Nucl Med 1991; 5:139 - 44.

[18] Sayed IS, Fong SY, Farhana M, Nurul Farizan M Z. 2016. Effects of Unconventional (Material) Filters on the Quality of Images Produced by Three Gamma Camera Systems in Tc-99m SPECT. International Journal of Healthcare Sciences 2016;4: 203-09.

[19] Sayed IS, Siti Zubaidah M. Use of an Effective Attenuation Coefficient Value and Material Filter Technique for Scatter Correction in Tc-99m SPECT. International Journal of Computing Academic Research 2016;5: 163-69. 